\title{
Optofluidic microscope and its applications in biology
}

\section{Xin Heng, Kevin W. Reynolds, Xiquan Cui, David Erickson, Demetri Psaltis, et al.}

Xin Heng, Kevin W. Reynolds, Xiquan Cui, David Erickson, Demetri Psaltis, Changhuei Yang, "Optofluidic microscope and its applications in biology," Proc. SPIE 6088, Imaging, Manipulation, and Analysis of Biomolecules, Cells, and Tissues IV, 608816 (21 February 2006); doi: 10.1117/12.640325

SPIE. Event: SPIE BiOS, 2006, San Jose, California, United States 


\title{
Optofluidic Microscope and its Applications in Biology
}

\author{
Xin Heng ${ }^{1}$, Kevin W. Reynolds ${ }^{2}$, Xiquan Cui ${ }^{1}$, David Erickson ${ }^{3}$, Demetri Psaltis ${ }^{1}$, Changhuei \\ Yang $^{1}$ \\ ${ }^{1}$ Department of Electrical Engineering, California Institute of Technology, Pasadena, CA, 91125; \\ ${ }^{2}$ Department of Physics, Norfolk State University, Norfolk, VA, 23504; ${ }^{3}$ Mechanical and Aerospace \\ Engineering, Cornell University, Ithaca, NY, 14853. \\ Email: $\underline{x i n @ \text { caltech.edu }}$
}

\begin{abstract}
:
In this article, we will demonstrate a novel optical imaging device that can be directly integrated into a microfluidic network, and therefore enables on-chip imaging in a microfluidic system. This micro imaging device, termed optofluidic microscope (OFM) is potentially free of bulk optics and is based on a nanohole array defined in a nontransmissive metallic layer that is patterned onto the floor of the microfluidic channel. The operation of the optofluidic microscope will be explained in details and its performance is examined by using a popular animal model, Caenorhabditis elegans (C. elegans). Images from a large population of nematode worms are efficiently acquired within a short time frame. The quality of the OFM images of C. elegans and the morphological characteristics revealed therein are evaluated. Two groups of early-stage $\mathrm{C}$ elegans larvae, wild-type and dpy-24 are successfully separated even though their morphological difference at the larval stage is subtle. The experimental results support our claim that the methodology described therein can be effectively used to develop a powerful tool for fulfilling high-resolution, highthroughput imaging task in microfluidics-based systems.
\end{abstract}

Keywords: Microfluidics, Nanophootnics, C elegans phenotyping

\section{INTRODUCTION}

Recently, there has been a rapid development in lab-on-a-chip devices that show improved performance by making use of numerous advantages of optical light. These advantages include the non-destructive nature of optical probing, the ease by which light field may be reconfigured, and the ability of light to 'walk through' transparent materials to manipulate objects. It is, therefore, not surprising that a lot of efforts have been recently directed towards the incorporation of optical tools into micro total analysis systems. Optical manipulation tools of this category include microfluidic sorter operated by optical force switching [1], optical tweezers in microfluidics [2], and all-optical microfluidic particle-sorter [3]. At the same time, various miniaturized optical probing and analyzing implementations have been realized with improved accuracy and reduced economical cost. These include micro fluorescence activated cell sorter [4], back scattering interferometry in rectangular channels [5], and numerous microscopy or fluorescence imaging tools applied to virus [6], DNA molecules [7, 8], single cells [9] and micro-organisms [10].

Although the microfluidic portion of these setups mentioned above is compact and cost effective, the optical components tend to be fairly complicated and occupy large space. There are numerous efforts presently underway to implement systems that seek to incorporate compact optical systems into microfluidics [11-14]. However, one particular on-chip instrumentation need has not been satisfactorily addressed, that is, on-chip optical imaging. High resolution imaging requirement in existing microfluidic systems is usually fulfilled by using bulky conventional microscopes, thus inevitably obviating the cost and size advantages of micro analysis systems. In this paper, we present a novel imaging technique, termed Optofluidic Microscopy (OFM), which enables high-resolution and high-throughput on-chip imaging. This is, to our knowledge, the first successful development of an on-chip imaging infrastructure that will have profound use in biological and clinical research.

Imaging, Manipulation, and Analysis of Biomolecules, Cells, and Tissues IV, edited by Daniel L. Farkas, Dan V. Nicolau, Robert C. Leif, Proc. of SPIE Vol. 6088, 608816, (2006) · 1605-7422/06/\$15 · doi: 10.1117/12.640325 


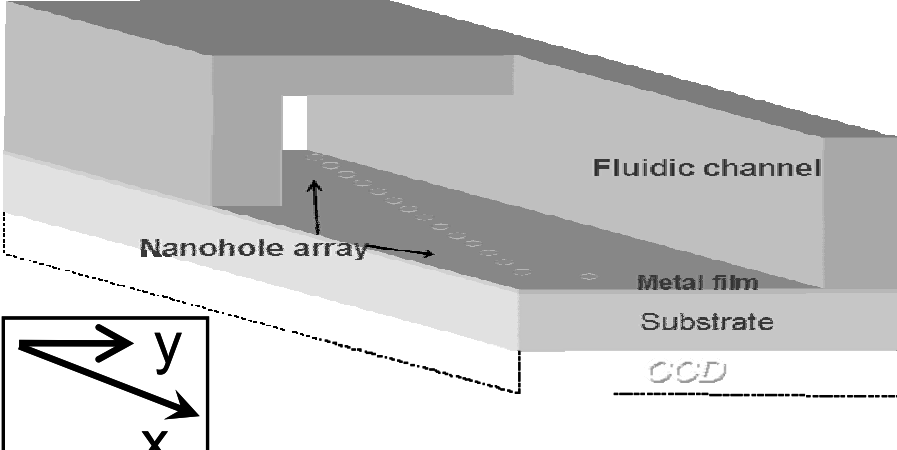

(a)

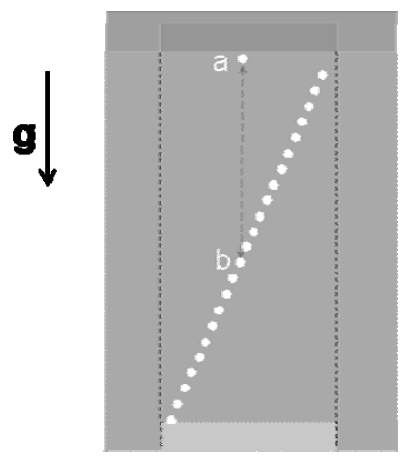

(b)
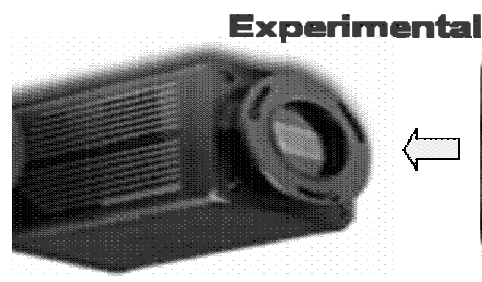

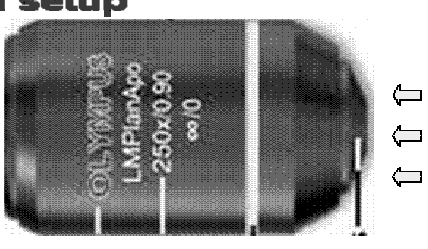

(c)

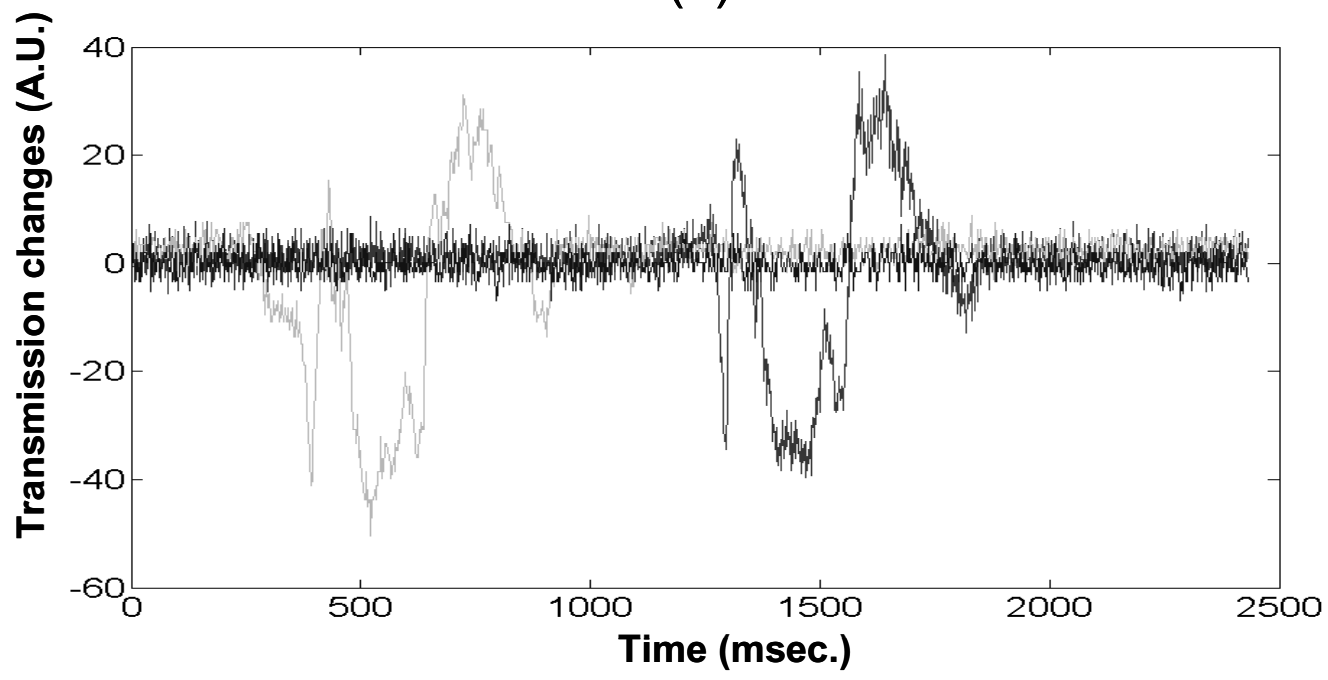

(d)

Figure 1: (a) 3D layout of OFM, where the microfluidic chip is hermetically bonded on top of the nanohole array layer; (b) view from the bottom: isolated hole [a] matches with the hole [b] in the nanohole array; g: orientation of gravitation; (c) Schematic representation of the current setup that includes a CCD array camera (Princeton Instruments Spec10-100), an inverted microscope (Olympus IX51) with a 40X objective lens (NA=0.65). (d) Signal time traces from three representative holes. Right peak (black): isolated hole; left peak (grey): isolated hole's corresponding hole

\section{METHODS}

The OFM device consists of a microfluidic chip that has a clear transmission ceiling and an optically absorptive floor made of metallic material (Fig. 1(a)). A slanted line of transmission holes are etched into the opaque 
metallic floor that is made of either aluminum or gold. In this prototype, the holes' diameter is about $600 \mathrm{~nm}$, and the optical absorptive layer is about $80 \mathrm{~nm}$ thick. Fabrication and alignment are detailed in Supplementary Materials. The device can then be mounted on top of a one dimensional CCD or photodiode array. For clarity, we shall define a coordinate system in relation to the O FM device. We set the $\mathrm{x}$-axis to be along the flow direction and the $\mathrm{y}$-axis to be normal to it (depicted in inset of Fig. 1(a)). Z-direction is normal to the device's plane. If we choose the inter-hole separation along the $\mathrm{x}$-direction to be equal to the CCD's pixel size, the transmission through each hole will map to individual pixel and thus will not interfere with each other. The determination of an object's shape and profile is accomplished by driving the object across the line of holes by any existing microfluidic delivery means. Notice that the system's pixel size along the $\mathrm{y}$-axis is set by the separation between the holes along $\mathrm{y}$-axis; and that along the $\mathrm{x}$-axis is equal to the ratio of the fluid flow speed and the pixel acquisition rate. The system resolution is by no means limited by the size of the pixels on the CCD array. The staggered-hole-array approach enables us to achieve high resolution $(\sim 600$ $\mathrm{nm}$ ) with commercial CCD linear arrays where the pixel size is typically $10 \mu \mathrm{m}$. Fabrication of the OFM prototype directly onto a sensor array is technically straightforward $[10,15]$. Therefore we concentrate on demonstrating the proof of concept and characterizing the performance of the optical system here. In our present demonstration, a conventional microscope is used in our experiments (Fig. 1 (c)). Transmission curves of individual holes are projected onto a CCD array (Princeton Instruments Spec-10 100) with the use of an Olympus IX51. The role of the microscope is simply to map each hole's transmission uniquely onto individual pixels on the CCD array.

The OFM device can be readily miniaturized, and is capable of acquiring high-resolution images of biological samples that are flowing in microfluidic channels. High-throughput imaging of individual biological samples can be efficiently acquired with a linear CCD array; the OFM images may then be used to perform phenotype sorting. Caenorhabditis elegans (C. elegans) is chosen as our first test organism to verify our system's capability to perform high-speed imaging and accurate evaluation of samples' morphological features.

\subsection{Experimental Results}

To demonstrate OFM's ability to perform imaging and phenotype characterization, we decided to focus on one particular application - to perform phenotype identification of a popular animal model - C elegans. C. elegans is a widely-used molecular genetic model for animal development, behavior and physiology. The molecular genetics has advanced to the point that genome-wide screens using libraries of RNAi have become standard; the determination of phenotypes is now the limiting step in genetic analyses. Furthermore, C. elegans are used for drug screening [16], but the phenotype determination involved is yet to be satisfactory. In many cases, body size and body shape are two major phenotypes. For example, abnormal TGF-beta signaling pathway affects body size [17]. However, in current C. elegans research, the parameters are usually qualitatively described by adjectives such as 'dumpy', 'small', 'slow', etc. This attributes to the fact that the difference between phenotypes often remains subtle to human observers. For all these reasons, automated and low cost phenotyping is crucial.

We used two different genotypes of nematodes at their first larval stage. At such an early stage, the geometrical difference among genotypes remains subtle to human eye observations. However, we will show later that highsensitivity OFM device is still able to separate them. We prepared the samples by first killing them in a 70C heat bath for 3 minutes. At the end of the process, the worms tend to be relaxed and straightened out. We then mix the worm with a $1 \%$ solution of Bovine Serum Albumin (BSA). The BSA solution helps the worms to more easily move in the micro channel. C elegans are transported through the channel by gravitational force, i.e. the microchip is vertically mounted. As each worm passes with a constant speed through the region that contains the slanted nanohole array, each individual hole will take a fine line scan over the sample in $\mathrm{x}$ direction. The time shift of the signals between adjacent holes will be a generic constant $(\tau)$ and thus can be easily corrected. Therefore we end up with a sequence of transmission time traces through individual nanoholes. To obtain the time-shift constant $\tau$, an isolated hole of the same size is drilled at either end of the nanohole array; the hole along the nanohole array that matches the isolated hole's y-axis displacement is then located (Fig. 1(b)). The time shift 
$\tau=\frac{\Delta x \times \Delta f r \times \delta t}{\Delta L}$

(1)

where $\Delta \mathrm{fr}$ is the difference of frame index between these two correspondent holes when the object flows across them, respectively; $\Delta \mathrm{x}$ is inter-hole separation projected in $\mathrm{x}$ direction; $\delta \mathrm{t}$ is camera's acquisition time (typically $2.5 \mathrm{msec}$ ) and $\Delta \mathrm{L}$ is the separation of those two correspondent holes. The pixel size in $\mathrm{x}$ direction $(\delta \mathrm{x})$ is determined as

$\delta x=\frac{\Delta x \times \delta t}{\tau}=\frac{\Delta L}{\Delta f r}$

The transmission time changes from two representative nanoholes are presented in Fig.1 (d). The profiles of the signals from two corresponding holes mentioned in the last paragraph are highly similar. It implies that this particular worm passed through the nanohole array without significant rotation. The rotated /tumbled samples are readily screened out by looking for the disparity between the two transmission signal traces.

C. elegans is one of the most studied model animals in biology due to its genetic tractability, completely sequenced genome, simple anatomy and body transparency. Studying varied nematode phenotypes has helped scientists to gain better understanding of signal transmission pathway in neurobiology, aging, mating and some other physiological behaviors. The difference between genotypes often remains subtle to human observers and there is a lack of smart imaging systems that can acquire quantitative data detailing their behavior or morphology in a labor saving manner. The optofluidic microscope uses the state of the art microfluidic technology to swiftly deliver biological organisms through the detection region (a chamber or a portion of fluidic channel) and image them, thus saving scientists a decent amount of time from having to measure one worm after another. For example, we have succeeded in getting data from more than 30 wild-type $C$ elegans larvae (L1) in around 1 minute. The extremely limited space occupied by fluidic channels (a few microns wide and tall) readily eliminates the potential troubles incurred by background interruption.

To demonstrate the capability of the OFM system in nematode phenotyping applications, we acquired OFM images from two separate populations of $C$. elegans: the wild-type, and a $d p y-24$ mutant which affects body morphology, i.e. the animals are expected to be slightly shorter and stouter than wildtype. The sample preparation procedure was identical. Manual measurements of body length and width were taken from the acquired images. The data

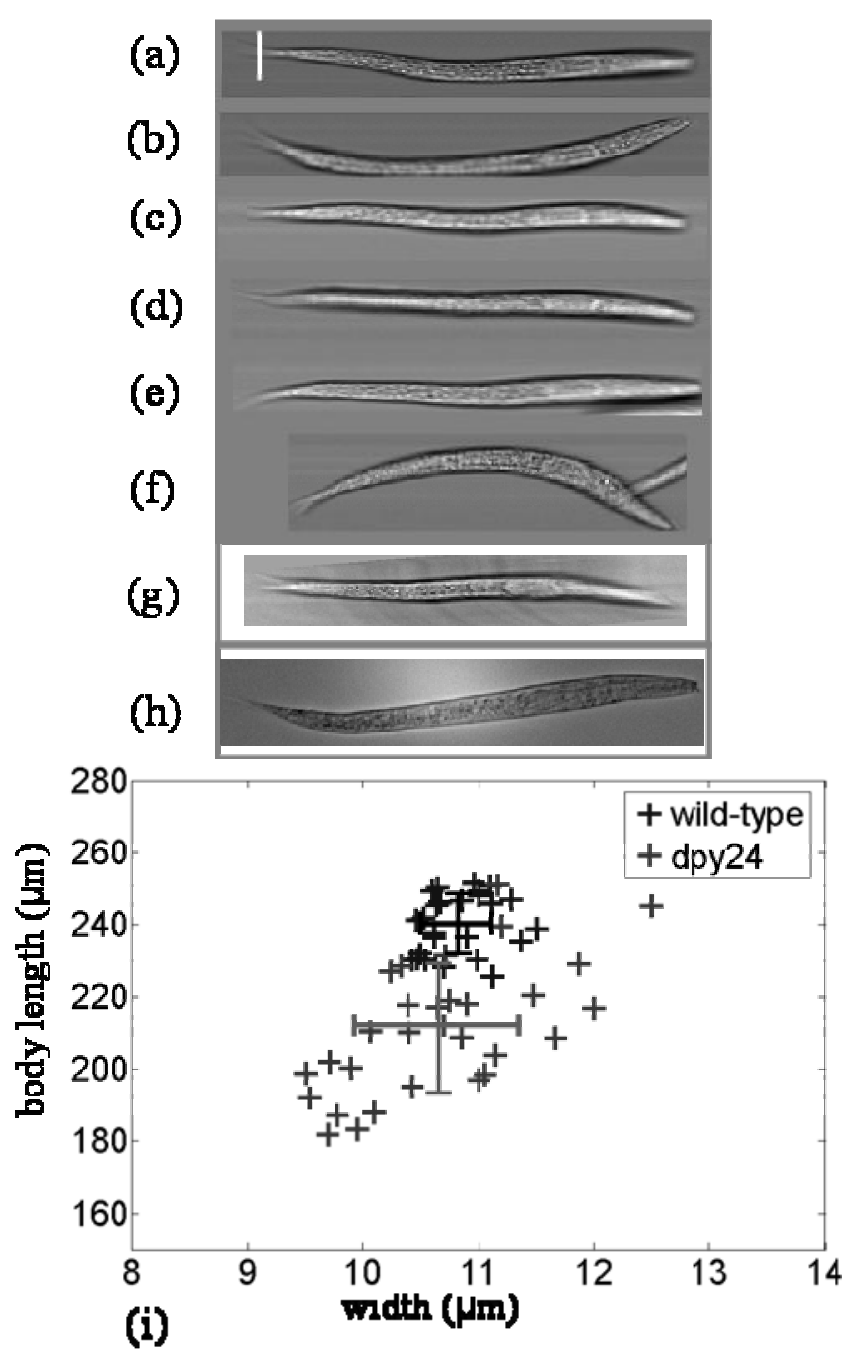

Figure 2 (a)-(f) Some OFM images of wild-type C. elegans larvae at the first larval stage; (g) OFM image of a dpy-24 mutant; note that the white bar represents $25 \mu \mathrm{m}$ for Fig.(a-g); (h) Conventional microscope image of a wild-type larva (not in the same scale as Fig.(a-g)); (i) Aspect ratio map of wild-type larvae (25 entities, black crosses); aspect ratio map of dpy-24 mutants (31 entities, grey crosses). The black srror bars and grey error bars represent the variance span (standard deviation) of the wild-type and the mutant, respectively. 
is plotted in an aspect-ratio map (Fig. 2(i)). Average length of wild-type larva is (240.1 \pm 8.3$) \mu \mathrm{m}$ and its average width is $(10.8 \pm 0.3) \mu \mathrm{m}$; this renders a body aspect-ratio of $(22.19 \pm 0.88)$. On the other hand, the average length of dpy 24 larva is $(211.2 \pm 17.9) \mu \mathrm{m}$ and its average width is $(10.7 \pm 0.7) \mu \mathrm{m}$; this gives an aspect ratio of $(19.84 \pm 1.35)$. The aspect-ratio map demonstrates a successful separation of these two phenotypes - specifically, we are able to distinguish two population sets of these two phenotypes from each other. Such image based analysis of phenotypes is similar to conventional microscopy based analysis. Our method is a significant improvement as it can be automated and the throughput is higher. This is the first time that high-resolution nematode imaging and high-throughput quantitative phenotyping are performed on a single microfluidic chip.

Some C. elegans flowed across the nanohole array in a 'nose-down' fashion, i.e. the front segments of the nematodes were in very close proximity to the nanohole array. The acquired images from these nematodes enable us to verify the achieved resolution of our OFM prototype. Fig. 3(a) shows one such image. By taking line scans across this image, we plotted the relationship of the location of the line scan and the sharpness of its body edge (Fig. 3(b)), where the sharpness is defined as the full width of the body edge that is presented in this image. The measurements from both ends of the worm body converge to $(710 \pm 50) \mathrm{nm}$. This measured resolution is very consistent with the predicted resolution of $600 \mathrm{~nm}$ as determined by the nanohole size.

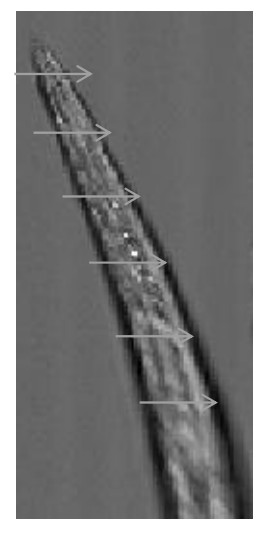

(a)

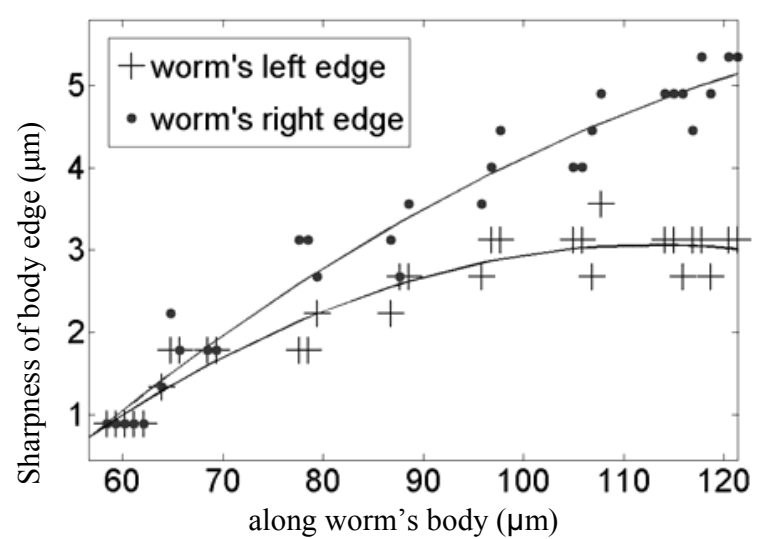

(b)

Figure 3 (a) OFM image of a 'nose-down' worm; the arrows represent the line scans along the worm's body; (b) Projection plot of the achieved resolution of the current OFM prototype; y-axis: the sharpness of the body edge; $x$-axis: the horizontal location of the line scan.

\subsection{Nanohole transmission}

Besides the experimental verification of the operation of OFM device, some preliminary calculations have been done with the focus on finding out the correlation of nanohole's spot size, filed distribution and the detection unit's sensitivity. Sensitivity of OFM device depends critically on the total transmission through each hole. Assuming the metal layer is perfectly conductive, two different regimes of hole diameter $(D)$ are examined.

$\boldsymbol{D}>>\lambda$, Large hole limit. In this regime, the diffraction of the hole matters little and the effective transmission area $A_{T}$ is simply equal to the physical cross section of the hole.

$D<<\lambda$, Small hole limit. In this regime and assuming that the film is infinitesimally thin, it was shown that the effective transmission area is given is proportional to the sixth power of the pinhole diameter in the leading order [18, 19]. In a recent EM simulation work, De Abajo [16] observed that the transmission is further attenuated exponentially as a function of the thickness of the ideal metal. Combining these two effects, we postulate that the effective transmission area can be expressed as:

$A_{T}=\left(\frac{16 \pi^{3}}{27}\right)\left(\frac{D^{6}}{\lambda^{4}}\right) \exp \left(-4 \pi d \sqrt{\frac{0.586^{2}}{D^{2}}-\frac{1}{\lambda^{2}}}\right)$

This formulation agrees fairly well with the simulation data that De Abajo [16] reported. In the study of the OFM's ultimate resolution, the detection system's signal to noise ratio needs being studied in more details. We adopt the 
standard photon counting model and assume that SNR $=1$ sets the lowest bound of the detectable signal that returns from the target. The total transmission photon count for a pixel dwell time $\tau$ (also equivalent to the inverse of frame rate) is given by,

$$
N_{T}=\frac{\varepsilon I A_{T} \tau}{h \frac{c}{\lambda}}
$$

where $h \frac{c}{\lambda}$ : the energy that one single photon carries; $I$ : illumination intensity; $\varepsilon$ : quantum efficiency of CCD camera.

The dominating noise source includes the photon counting noise (shot noise) and the receiver noise $\left(n_{r} \tau\right)$. Thus the sensitivity (SNR, or signal to noise ratio) can be expressed as:

$$
S N R=\frac{N_{T}}{\sqrt{N_{T}+\left(n_{r} \tau\right)^{2}}}
$$

Therefore, biological imaging with a micron level resolution and $30 \mathrm{~dB}$ sensitivity can be readily performed with the use of natural light illuminations. In principal, sub-wavelength resolution can be achieved in an OFM device by simply spacing the adjacent holes in the $y$ direction that matches up with the desired resolution limit. As the holes are well separated in $\mathrm{x}$-direction, their transmission contributions will be distinguishable from each other on a CCD camera. The state of the art nanofabrication technology enables the creation of etching patterns with resolution of 10's of nanometer. Therefore, the existing fabrication technique will not stop us from creating OFM devices with resolution of sub-100 nanometers. Our preliminary estimates based on Equations (3)-(5) shown in graphical forms in Fig. 4 predict that with $600 \mathrm{~nm}$ laser illumination, achieving $40 \mathrm{~dB}$ signal sensitivity for OFM with resolution of $100 \mathrm{~nm}$ is highly possible. This achievable resolution will break the diffraction limit and thus add more fine information into the produced images that can not be achieved by using conventional microscope.

\section{CONCLUSION AND FUTURE WORK}

We have presented what to our knowledge is the first effort at integrating a microscope into a single biochip. We have demonstrated that OFM holds much promise of being a reliable and efficient on-chip microscopy tool with high resolution. The nano-aperture array in OFM functions as an array of optical probes, which is a novel configuration different from other existing microscopy techniques. High resolution is highly possible with smaller nano apertures with engineered shapes in favor of enhanced transmission. The images OFM from $\mathrm{C}$ elegans of different phenotypes show 
well distinguishable morphological features that were time-consuming and difficult to obtain by direct human observation.

The utility of the OFM systems for C. elegans research extends beyond phenotypic characterization. For example, the system could be modified to include real-time phenotype sorting, and it is possible to build a closed-loop lab-on-a-chip system that can incubate and perform automated imaging of a single nematode for life-cycle studies (e.g. a few days). Also, the automated nature and accuracy of the OFM method implies we can use it to measure relatively subtle phenotypic variation within a population of one phenotype. Quantitative analysis of phenotypic variance is likely to be insightful in studying the effects of environment stress, nutrition and drugs on growth, reproduction, and life span of C. elegans.

In addition to nematode related research, the OFM can also be used to image any micro-organisms or biological cells that can be suspended in a liquid medium. Potential applications include blood fraction analysis [20], urine screening for infection [21, 22], stem cells screening and sorting[23, 24], and tumor cell counting [25, 26]. Additional functionalities can be built into the OFM systems. For example, the capability to perform fluorescence imaging [15, 27] or Raman imaging [28] can be added In fact, the on-chip nature of the OFM easily enables multispectral analysis. A series of OFM device, each selective for a given wavelength band, can be built along a single microfluidic channel. The simplicity and compact nature of OFM systems, and the fact that they can be created with existing fabrication technology will translate to their swift adaptation as an important lab-on-a-chip component.

\section{SUPPLEMENTARY MATERIALS}

\section{Chip fabrication and alignment:}

The nanohole array is defined in non-transmissive metal foil, i.e. gold or aluminum. In the fabrication of nano array in gold, holes are directly made by focused ion beam (FIB) on a 80nm thick gold film, which is deposited on ultra-clean quartz wafer with chromium as the adhesion layer material; in the fabrication of nano array in aluminum, we deposit a 90-nanometer layer of Al on clean quartz wafer followed by spinning a 200nm layer of PMMA on top of it. Next, the nanohole array is patterned on PMMA by using high resolution electron beam writer, and later transferred into aluminum by using dry ion beam etching. In the prototype used in this demonstration experiments, the diameter of the nanohole is all about $600 \mathrm{~nm}$. The single-layer PDMS microfluidic chip is produced by standard replica molding. In

light of the present application purpose, the channel cross section is about $30 \mu \mathrm{m}$ wide and $20 \mu \mathrm{m}$ tall. The alignment of the fluidic layer and the metallic layer is accomplished by using an inverted microscope or a Karl Suss mask aligner. Afterwards, the alignment can be verified by using an upright microscope.

\section{ACKNOWLEDGEMENT}

We are grateful for the generous help from Dr. Larry R. Baugh and Professor Sternberg regarding C elegans culturing and phenotyping, and the help from Professor Axel Scherer, Dr. Joerg Schilling in fabrication. We thank Dr. Zahid Yaqoob, Jigang Wu for constructive discussions. The assistance from Cornell Nanofabrication Facility and Caltech Watson cleanroom is appreciated. This project is funded by DARPA's center for optofluidic integration

\section{REFERENCE}

[1] M. M. Wang, E. Tu, D. E. Raymond, J. M. Yang, H. C. Zhang, N. Hagen, B. Dees, E. M. Mercer, A. H. Forster, I. Kariv, P. J. Marchand, and W. F. Butler, "Microfluidic sorting of mammalian cells by optical force switching," Nature Biotechnology, vol. 23, pp. 83-87, 2005.

[2] D. G. Grier, "A revolution in optical manipulation," Nature, vol. 424, pp. 810-816, 2003. 
[3] M. P. MacDonald, G. C. Spalding, and K. Dholakia, "Microfluidic sorting in an optical lattice," Nature, vol. 426, pp. 421-424, 2003.

[4] A. Y. Fu, C. Spence, A. Scherer, F. H. Arnold, and S. R. Quake, "A microfabricated fluorescence-activated cell sorter," Nature Biotechnology, vol. 17, pp. 1109-1111, 1999.

[5] D. A. Markov, K. Swinney, and D. J. Bornhop, "Label-free molecular interaction determinations with nanoscale interferometry," Journal of the American Chemical Society, vol. 126, pp. 16659-16664, 2004.

[6] D. Akin, H. B. Li, and R. Bashir, "Real-time virus trapping and fluorescent imaging in microfluidic devices," Nano Letters, vol. 4, pp. 257-259, 2004.

[7] S. Mangenot, M. Hochrein, J. Radler, and L. Letellier, "Real-time imaging of DNA ejection from single phage particles," Current Biology, vol. 15, pp. 430-435, 2005.

[8] J. O. Tegenfeldt, O. Bakajin, C. F. Chou, S. S. Chan, R. Austin, W. Fann, L. Liou, E. Chan, T. Duke, and E. C. Cox, "Near-field scanner for moving molecules," Physical review letters, vol. 86, pp. 1378-1381, 2001.

[9] P. C. H. Li, L. de Camprieu, J. Cai, and M. Sangar, "Transport, retention and fluorescent measurement of single biological cells studied in microfluidic chips," Lab on a Chip, vol. 4, pp. 174-180, 2004.

[10] D. Lange, C. W. Storment, C. A. Conley, and G. T. A. Kovacs, "A microfluidic shadow imaging system for the study of the nematode Caenorhabditis elegans in space," Sensors and Actuators B-Chemical, vol. 107, pp. 904914, 2005.

[11] J. Koschwanez, M. Holl, B. Marquardt, J. Dragavon, L. Burgess, and D. Meldrum, "Identification of budding yeast using a fiber-optic imaging bundle," Review of Scientific Instruments, vol. 75, pp. 1363-1365, 2004.

[12] J. M. Ruano, V. Benoit, J. S. Aitchison, and J. M. Cooper, "Flame hydrolysis deposition of glass on silicon for the integration of optical and microfluidic devices," Analytical Chemistry, vol. 72, pp. 1093-1097, 2000.

[13] J. P. Valentino, S. M. Troian, and S. Wagner, "Microfluidic detection and analysis by integration of thermocapillary actuation with a thin-film optical waveguide," Applied Physics Letters, vol. 86, 2005.

[14] D. Y. Zhang, N. Justis, and Y. H. Lo, "Integrated fluidic lenses and optic systems," Ieee Journal of Selected Topics in Quantum Electronics, vol. 11, pp. 97-106, 2005.

[15] M. L. Adams, M. Enzelberger, S. Quake, and A. Scherer, "Microfluidic integration on detector arrays for absorption and fluorescence micro-spectrometers," Sensors and Actuators a-Physical, vol. 104, pp. 25-31, 2003.

[16] K. Evason, C. Huang, I. Yamben, D. Covey, and K. Kornfeld, "Anticonvulsant medications extend worm lifespan.," Science, vol. 307, pp. 258-262, 2005.

[17] C. Savage-Dunn, "TGF- $\beta$ signaling, WormBook, ed. The C. elegans Research Community, WormBook,doi/10.1895/wormbook.1.22.1, ," http://www.wormbook.org, 2005.

[18] H. A. Bethe, "Theory of diffraction by small holes," Physical Review, vol. 66, pp. 163-182, 1944.

[19] C. J. Bouwkamp, "Diffraction theory," Reports on Progress in Physics XVIII, pp. 35, 1954.

[20] W. Cui, W. Cui, Y. Fan, W. Wu, F. Zhang, J. Y. Wang, A. P. Ni, and "Expression of lymphocytes and lymphocyte subsets in patients with severe acute respiratory syndrome," Clinical infectious diseases, vol. 37, pp. 857-859, 2003.

[21] J. M. Marrazzo, C. L. White, B. Krekeler, C. L. Celum, W. E. Lafferty, W. E. Stamm, H. H. Handsfield, and "Community-based urine screening for Chlamydia trachomatis with a ligase chain reaction assay," Annals of internal medicine, vol. 127, pp. 796, 1997.

[22] K. C. Carroll, D. C. HALE, D. H. VONBOERUM, G. C. REICH, L. T. HAMILTON, J. M. MATSEN, and "LABORATORY EVALUATION OF URINARY-TRACT INFECTIONS IN AN AMBULATORY CLINIC," American journal of clinical pathology, vol. 101, pp. 100-103, 1994.

[23] G. B. McCowage, K. L. Phillips, T. L. Gentry, S. Hull, J. Kurtzberg, E. Gilboa, C. Smith, and "Multiparameter-fluorescence activated cell sorting analysis of retroviral vector gene transfer into primitive umbilical cord blood cells," Experimental hematology, vol. 26, pp. 288-298, 1998.

[24] J. Buchstaller, L. Sommer, M. Bodmer, R. Hoffmann, U. Suter, N. Mantei, and "Efficient isolation and gene expression profiling of small numbers of neural crest stem cells and developing Schwann cells," The journal of neuroscience, vol. 24, pp. 2357-2365, 2004.

[25] T. Fujiwara, D. W. CAI, R. N. GEORGES, T. MUKHOPADHYAY, E. A. GRIMM, and J. A. ROTH, "THERAPEUTIC EFFECT OF A RETROVIRAL WILD-TYPE P53 EXPRESSION VECTOR IN AN ORTHOTOPIC LUNG-CANCER MODEL," Journal of the National Cancer Institute, vol. 86, pp. 1458-1462, 1994. 
[26] S. B. Fox, K. C. GATTER, R. BICKNELL, J. J. GOING, P. STANTON, T. G. COOKE, A. L. HARRIS, and "RELATIONSHIP OF ENDOTHELIAL-CELL PROLIFERATION TO TUMOR VASCULARITY IN HUMAN BREAST-CANCER," Cancer research, vol. 53, pp. 4161-4163, 1993.

[27] H. W. Gai, Y. Li, Z. Silber-Li, Y. F. Ma, and B. C. Lin, "Simultaneous measurements of the flow velocities in a microchannel by wide/evanescent field illuminations with particle/single molecules," Lab on a Chip, vol. 5, pp. 443-449, 2005.

[28] J. B. Salmon, A. Ajdari, P. Tabeling, L. Servant, D. Talaga, M. Joanicot, and "In situ Raman imaging of interdiffusion in a microchannel," Applied physics letters, vol. 86, 2005. 